\title{
Effects of static and dynamic image noise and background luminance on letter contrast threshold
}

\author{
Asli Kumcu, Ljiljana Platiša, and Wilfried Philips \\ iMinds-TELIN-IPI, Ghent University \\ Ghent, Belgium \\ Email: akumcu@telin.ugent.be
}

\begin{abstract}
We performed a pilot psychovisual experiment to determine the contrast threshold and slope of the psychometric function for a target embedded in two levels of static and dynamic external image noise. Sloan letters were presented in a local background surrounded by a global background, both varied over four luminance levels: $\mathbf{5 8 . 6 2}, \mathbf{1 5 5 . 9 7 ,} \mathbf{2 5 3 . 5 0}$, and 347.47 candela per square meter. Uncorrelated Gaussian noise with normalized standard deviation 0.019 and 0.087 was added to the stimuli. A noise-free stimulus was also tested. No systematic effect of global background luminance was found. The contrast threshold was approximately $1 \%$ in the noise-free stimulus and increased monotonically with rms noise contrast, following a power law relationship. Thresholds were higher in static noise. The model will be incorporated in a no-reference, task-based medical quality metric for $x$-ray sequences.
\end{abstract}

\section{INTRODUCTION}

In medical imaging, the ability to conduct a clinical task usually depends on the visibility of clinically relevant targets (objects) in the image. Target detectability is strongly influenced by the contrast ratio between the target and the background as well as image noise. In applications such as interventional X-ray, in which contrast medium-filled blood vessels (the target) are visualized as a sequence of frames at a fast frame rate, dynamic image noise may mask the target by a different amount than static noise in single frames. Visibility may also be affected by the background intensity, which varies by anatomical location, patient thickness, and projection angle. Existing methods for assessing signal detection performance in medical images, called model observers, can estimate the visibility of clinical targets in multislice images [1] but require extensive training to the image data. Our approach is to design a real-time, task-based quality measure that does not require training. The measure estimates the probability of detecting a target using a psychometric model of contrast detection measured for three image features: background intensity, noise, and frame rate. While estimates of contrast sensitivity as a function of image noise and background can be found in the literature, we found no reports of the slope of the psychometric curve for dynamic noise. Therefore, the main contribution of this work is to measure and characterize the entire psychometric curve for dynamic noise. We conducted a pilot psychovisual experiment to determine the psychometric

This work was performed within the PANORAMA project (co-funded by grants from Belgium, Italy, France, the Netherlands, the United Kingdom, and the ENIAC Joint Undertaking). We thank the anonymous reviewers for their helpful comments. curve of contrast detection as a function of local and global background, noise variance, and static versus dynamic noise.

\section{METHODS}

Experiments and analysis were conducted with PsychoPy [2] version v1.78.01. Stimuli were presented to two observers at $50 \mathrm{~cm}$ viewing distance on a linearized medical LCD color display (MDCC-3120-DL, Barco, Kortrijk, Belgium), 2048×1536 pixels, 30-bit color, $25 \mathrm{Hertz}$, set to native white diagnostic mode, minimum and maximum luminance 0.78 and 389.5 candela per square meter $\left(\mathrm{cd} / \mathrm{m}^{2}\right)$. A contrast detection task was conducted with a 1 up/1 down single stimulus staircase procedure with two interleaved staircases. The target was one of nine letters (VRSKDNHOZ) in Sloan font [3] selected at random for each trial, displayed at $0.5^{\circ}$ height (stroke frequency of 3.2 cycles per degree (cpd) [4]) in the center of the display. Sloan letters were selected because their properties have been well studied in the literature and may be more representative of clinical targets such as vessels than Gabor patches. At maximum contrast, the target was approximately $0.78 \mathrm{~cd} / \mathrm{m}^{2}$, representing a contrast medium-filled vessel. An anchor letter was displayed $2.5^{\circ}$ to the top-right to facilitate letter identification. The background was a circle of diameter $5.88^{\circ}$ (256 pixels), considered the local background, surrounded by a square of $11.76^{\circ}$ (512 pixels) height and width, considered the global background. Backgrounds were displayed at four values approximately equally spanning the display's luminance range: 58.62, 155.97, 253.50, and 347.47 $\mathrm{cd} / \mathrm{m}^{2}$. Two noise types and two noise levels were added to the target and background. The noise type was static or dynamic, where static noise was a fixed 2D noise field, and dynamic noise was a sequence of independent $2 \mathrm{D}$ noise fields and viewed at 25 frames per second. Uncorrelated Gaussian noise of unit normalized standard deviation $\sigma_{1}=0.019, \sigma_{2}=0.087$ was added to the images to simulate noise levels in high and low dose interventional x-ray sequences. A static noisefree condition was also tested. No viewing time limit was imposed. Eighty conditions were tested as a combination of four local and four global backgrounds, two noise levels and two noise types, and a noise-free condition. See Fig. 1(a) for two examples.

The contrast of the target was estimated as the Weber contrast: $C_{\mathrm{T}}=\left(L_{\mathrm{LB}}-L_{\mathrm{T}}\right) / L_{\mathrm{LB}}$ where $L_{\mathrm{LB}}$ is the mean local background luminance and $L_{\mathrm{T}}$ is the target's mean luminance. Noise was also expressed as rms noise contrast [5]: $C_{\mathrm{RMS}}=\sigma / L_{\mathrm{LB}}$ with both terms in luminance units. The slope 


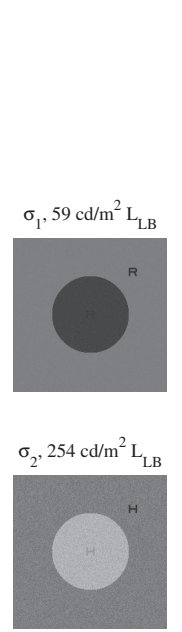

(a) Two stimuli



(b) Psychometric curves for contrast detectability
Fig. 1. (a) Two stimuli with approximately the same contrast threshold (5\% and 3\% respectively for static and dynamic noise) and similar rms noise contrast: 0.126 for top image, 0.133 for bottom image. (b) Psychometric curves, pooled across observers and global backgrounds. One plot per noise level and noise type. Local background luminance indicated by color.

and location of the contrast ratio psychometric curve for each condition was determined by fitting the staircase results per observer to a two-parameter Weibull function. The contrast threshold $T$ was determined at the $50 \%$ detection probability level, or percent correct (PC), by pooling across observers and global backgrounds, and fit to $C_{\mathrm{RMS}}$.

\section{RESULTS}

For static noise $\sigma_{2}$ at $58.62 \mathrm{~cd} / \mathrm{m}^{2}$, the staircases for one global background for observer 1 and three global background levels for observer 2 did not converge. No systematic effect of global background luminance was found. Therefore, the psychometric curves were pooled and are shown in Fig. 1(b). In all noisy conditions, the psychometric curve was flatter in darker background, at the higher noise level, and in static images. For some conditions, such as static noise $\sigma_{2}$, the slope did not decrease monotonically with background luminance.

Contrast thresholds at $50 \%$ PC for the noise-free stimulus were $1.57 \%$ at $58.62 \mathrm{~cd} / \mathrm{m}^{2}$ and approximately $1 \%$ for the other local backgrounds. For the noise stimuli, contrast threshold increased monotonically with background luminance, noise level, and $C_{\mathrm{RMS}}$, but more so in static noise. The relationship was best modeled with a power law function: $T_{\text {Dynamic }}=$ $0.137 x^{0.743}$ and $T_{\text {Static }}=0.188 x^{0.663}$ where $x$ is $C_{\mathrm{RMS}}$.

\section{DiscusSiON \& CONCLUSION}

The results from this pilot study indicated an effect of noise type (static or dynamic), noise variance, and local background luminance on contrast threshold and the slope of the psychometric curve, but no measurable effect of global background. While contrast threshold at 50\% PC increased monotonically with $C_{\mathrm{RMS}}$, the slope of the psychometric curve did not for some conditions. In addition, several of the staircases at the lowest background luminance level did not converge. This may potentially have been due to the difficulty of detecting a dark target on a dark background. An adaptive n-alternative forced choice (nAFC) procedure may allow us to more accurately measure the full psychometric curve, especially for conditions where the observer's uncertainty has been shown to be high, such as in dark backgrounds and high noise levels. For the noise-free condition, contrast threshold was approximately $1 \%$, roughly in line with [5] and [6]. In noisy stimuli, contrast threshold increased with a power law relationship to $C_{\mathrm{RMS}}$ and was higher in static noise, consistent with [6]. Contrast thresholds measured in this study were approximately 2 to 2.5 times lower than [6] at equivalent $C_{\mathrm{RMS}}$ levels for $3.2 \mathrm{cpd}$ Gabor patches with added static and dynamic uniform noise in synchronous mode; our lower thresholds may partially be explained by the PC target: we targeted 50\% PC whereas [6] targeted $82 \%$ PC. In addition, the 25 millisecond response time of the LCD monitor used in our study, compared the nearly negligible response time for a CRT (used in [6]), may have slightly reduced the displayed noise level. Compared to static noise added to gratings in [5], our contrast threshold at 0.02 $C_{\mathrm{RMS}}$ is nearly double that of [5], but only $13 \%$ higher at $0.1 C_{\mathrm{RMS}}$, even though they targeted $75 \% \mathrm{PC}$. In contrast to our study, [5] reports a logarithmic relationship between $C_{\mathrm{RMS}}$ and contrast threshold. Differences in stimuli presentation may partially account for these differences - both studies present the stimuli for durations of 1 second or less. In addition, we vary $C_{\mathrm{RMS}}$ as a function of background luminance as well as noise variance, whereas [5] and [6] fix background luminance and vary noise level.

The main contribution of this work is to characterize the influence of background luminance and noise level on the psychometric curve for sequences of noisy images viewed in dynamic mode. This model of contrast detectability will serve as the basis for a task-based video quality metric for medical $\mathrm{X}$-ray sequences, such as interventional X-ray imaging. We plan to conduct a follow-up study to estimate the slope of the psychometric curve more accurately, in order to model detectability of targets in dynamic noise as a function of contrast ratio, noise level, and background intensity. We also plan to incorporate the motion of the target into the detectability model, as moving targets may be easier to detect at certain velocities.

\section{REFERENCES}

[1] L. Platiša, B. Goossens, E. Vansteenkiste, S. Park, B. Gallas, A. Badano, and W. Philips, "Channelized hotelling observers for the assessment of volumetric imaging data sets," J. Opt. Soc. Am. A, vol. 28, no. 6, pp. 1145-1163, Jun 2011.

[2] J. W. Peirce, "PsychoPy - psychophysics software in Python," Journal of Neuroscience Methods, vol. 162, no. 1-2, pp. 8-13, 2007.

[3] D. G. Pelli, J. G. Robson, and A. J. Wilkins, "The design of a new letter chart for measuring contrast sensitivity," Clinical Vision Sciences, pp. 187-199, 1988.

[4] N. J. Majaj, D. G. Pelli, P. Kurshan, and M. Palomares, "The role of spatial frequency channels in letter identification," Vision Research, vol. 42, no. 9, pp. $1165-1184,2002$.

[5] J. P. Thomas, "Effect of static-noise and grating masks on detection and identification of grating targets," J. Opt. Soc. Am. A, vol. 2, no. 9, pp. 1586-1592, Sep 1985.

[6] J. J. McAnany and K. R. Alexander, "Spatial contrast sensitivity in dynamic and static additive luminance noise," Vision Research, vol. 50, no. 19 , pp. 1957 - 1965, 2010 . 